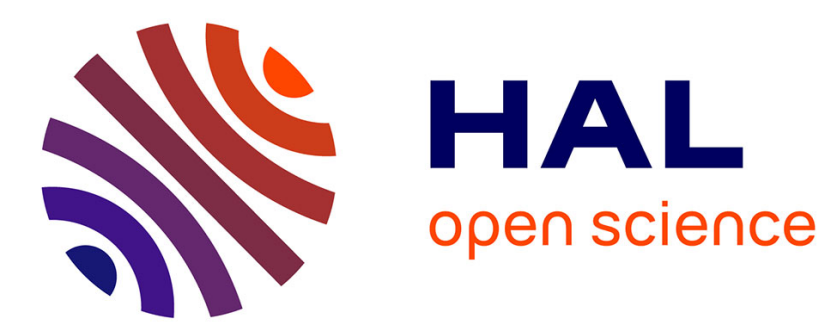

\title{
The kink chain revisited
}

Gunther Schoeck

\section{To cite this version:}

Gunther Schoeck. The kink chain revisited. Philosophical Magazine, 2007, 87 (11), pp.1631-1647. 10.1080/14786430600788954 . hal-00513715

\section{HAL Id: hal-00513715 https://hal.science/hal-00513715}

Submitted on 1 Sep 2010

HAL is a multi-disciplinary open access archive for the deposit and dissemination of scientific research documents, whether they are published or not. The documents may come from teaching and research institutions in France or abroad, or from public or private research centers.
L'archive ouverte pluridisciplinaire HAL, est destinée au dépôt et à la diffusion de documents scientifiques de niveau recherche, publiés ou non, émanant des établissements d'enseignement et de recherche français ou étrangers, des laboratoires publics ou privés. 


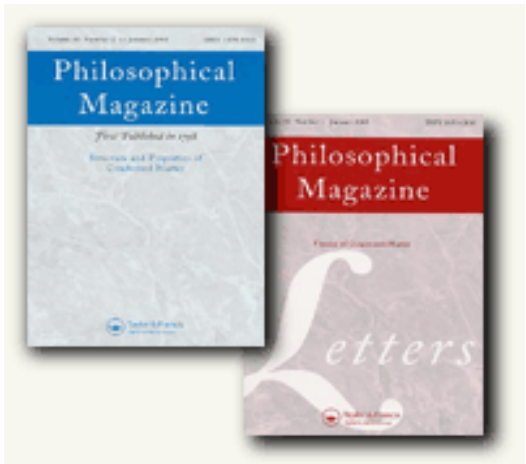

\section{The kink chain revisited}

\begin{tabular}{|r|l|}
\hline Journal: & Philosophical Magazine \& Philosophical Magazine Letters \\
\hline Manuscript ID: & TPHM-06-Feb-0032.R1 \\
\hline Journal Selection: & Philosophical Magazine \\
\hline Date Submitted by the \\
Author: & 06-Apr-2006 \\
\hline Complete List of Authors: & $\begin{array}{l}\text { Schoeck, Gunther; University of Vienna, Institute of Materials } \\
\text { Physics }\end{array}$ \\
\hline Keywords: & dislocation theory, numerical modelling \\
\hline Keywords (user supplied): & kinks \\
\hline &
\end{tabular}

\section{s ScholarONE" \\ Manuscript Central}


Revised version.

\title{
The kink chain revisited
}

by

\author{
Gunther Schoeck \\ Institute of Materials Physics, University of Vienna, Boltzmanng.5,1090 Vienna, Austria \\ E-mail: gunther.schoeck@univie.ac.at
}

\begin{abstract}
The equilibrium configuration of a kink chain consisting out of kink pairs, formed in a dislocation segment bowing out under the action of an applied stress $\sigma$ is determined, by deriving an expression for the enthalpy $\mathrm{H}(\sigma)$, taking account of all kink-kink interactions. For a realistic description, instead of the usual $1 / \mathrm{r}$ asymptotic potential, the Püschl potential must be used, which does not diverge when two kinks of the same sign approach each other. For a given applied stress $\sigma$ above a critical stress, there exist, due to the geometrical restraints, a number of different bow-out configurations with different numbers of kink pairs and the problem is to find the 'ground state', which is the state with the lowest enthalpy. This can only be done by numerical or semi-graphical methods. Since the ground state can only be reached by thermal activation, there is an asymmetry in the forward and backward movement. It is found that in the equilibrium configuration the kinks of same sign generally accumulate at the two opposite side arms, leaving a longer straight segment in-between. When the bowout height of the kink chain approaches the height resulting from the line tension model, the influence of the long-range back-stress caused by the overall curvature cannot be neglected and it limits the accessible height.
\end{abstract}

\section{Introduction}

Straight dislocation lines in crystalline solids running along close-packed lattice directions experience upon displacement small periodic variations in the line energy, the Peierls energy $[1,2]$. This energy variation results from a change in the arrangement of the atoms in the core region, when the dislocation is displaced in a crystal. The periodicity is equal to the lattice row separation $a$. In equilibrium without applied stress the dislocation lines lie along the bottom of the Peierls valleys. They can cross over the Peierls ridges into the neighbouring Peierls valley in a short segment called kink, a concept introduced by Shockley [3]. The 
extension or width $\mathrm{w}$ of the kink is controlled by the balance between the elastic energy or line tension $\mathrm{T}_{\mathrm{L}}$, which wants to make it wide, and the atomic misfit energy or Peierls energy $\mathrm{E}_{\mathrm{P}}$, which wants to make it short. Based on the line tension approach the configuration $\mathrm{y}(\mathrm{x})$ for a kink in a dislocation along the $\mathrm{x}$-axis in a sinusoidal Peierls potential with Peierls energy $\mathrm{E}_{\mathrm{P}}$ is described $[4,5]$ by

$$
\mathrm{y}(\mathrm{x})=\frac{2 \mathrm{a}}{\pi} \operatorname{arctg}\left(\exp \left(\frac{\pi \mathrm{x}}{\mathrm{w}_{0}}\right)\right)
$$

with width $\mathrm{w}_{0}=\mathrm{a} \sqrt{\mathrm{T}_{\mathrm{L}} / 2 \mathrm{E}_{\mathrm{P}}}$. Here $\mathrm{T}_{\mathrm{L}}=\hat{\mathrm{T}}_{\mathrm{L}} \ln \left(\Lambda / \mathrm{r}_{0}\right)$ is the line tension with pre-exponential line tension factor $\hat{T}_{L}=E_{L}+d^{2} E_{L} / d \varphi^{2}$ for a dislocation of orientation $\varphi$ with line energy $E_{L} \cdot \Lambda$ and $r_{0}$ are the 'appropriate' somewhat ill defined outer and inner cut-off radii. Within the framework of this approximation the energy of a kink is

$$
\mathrm{E}_{\mathrm{K}}=\frac{\mathrm{a}}{\pi^{2}} \sqrt{\mathrm{E}_{\mathrm{P}} \mathrm{T}_{\mathrm{L}}}
$$

The kinks can have opposite directions (up and down) also called opposite signs, where the assignment of the \pm sign is arbitrary. Originally it was thought, that there is only a short-range interaction between kinks, but as found by Kroupa and Brown [6], Seeger and Schiller [5] and Eshelby [7] there is also a long-range interaction. The long-range interaction potential $\mathrm{U}_{\mathrm{KK}}$ for abrupt kinks (with w=0), or for kinks of height $a$ for which the separation $r$ is large compared to the kink width $w$, is given by

$$
\mathrm{U}_{\mathrm{KK}}= \pm \frac{\hat{\mathrm{T}}_{\mathrm{L}} \mathrm{a}^{2}}{2 \mathrm{r}}
$$

where the + sign applied for kinks of the same sign and the - sign for kinks of opposite sign.

When a dislocation segment of length $\mathrm{L}$ is oriented at a small angle $\varphi$ against the Peierls valley and anchored $\mathrm{N}$ valleys apart, it will contain $\mathrm{N}$ geometrical kinks, which cross over the intermediate Peierls ridges. The thermodynamic properties of such a kin-chain have been studied by Seeger and Schiller [5, 8] and Alefeld [9]. Considering only nearest neighbour interaction, the kinks originally are equally spaced with a separation $\mathrm{d}_{0}=\mathrm{L} /(\mathrm{N}-1)$. Under the action of a resolved shear stress $\sigma$, the segment will bow out by lateral displacement of the kinks and the separation between kink $n$ and $n+1$ is given by Lems [10] as

$$
\mathrm{d}_{\mathrm{n}}=\frac{\mathrm{d}_{1}}{\sqrt{1-(\mathrm{n}-1)\left(\sigma \mathrm{ba} / \hat{\mathrm{T}}_{\mathrm{L}}\right) \mathrm{d}_{1}^{2}}},
$$

where $d_{1}$ is the separation between the two leading kinks and is determined by the condition 


$$
\sum_{n=1}^{N-1} d_{n}=L
$$

When also the long-range interaction between the different kinks is accounted for, the configuration without applied stress will be S-shaped, since due to the mutual repulsion the kinks pile up at the opposite anchoring points. The deviation from a straight line is however small.

Of more interest and of considerable more complexity is the behaviour of a segment lying originally along a Peierls valley, which has bowed out by the formation of kink pairs under the action of an applied stress. The equilibrium configuration of such a kink chain has not yet been determined in a satisfactory way, since the evaluation of the analytic expressions and the minimizing procedure can only be done numerically. It is the subject of the present paper. There are treatments in the literature, which obtained the configuration using a modified line tension approach $[11,12,13]$, by including the action of the periodic Peierls stress. Since this is a strictly local approximation, it cannot adequately take account of the long-range interaction between the kinks. The solution of the problem is not only of academic interest, in closing a gap in our knowledge, but also essential in answering the question: which magnitude of the Peierls energy is requested, that long segments of dislocations remain straight - as for instance observed in $\mathrm{Si}$ - in spite of being under the action of an internal stress.

When trying to find the equilibrium configuration, it turns out, that the asymptotic potential $\mathrm{U}_{\mathrm{KK}}$ given by eq. 2 cannot adequately describe the kink-kink interaction in the present situation, since it diverges when two kinks approach each other. We will use therefore here a kink pair potential $\mathrm{V}_{\mathrm{KK}}$ derived by Püschl et al. [14], which takes account of the finite width $\mathrm{w}$ of the kinks and which approaches the energy of a kink twice the height, when two kinks of the same sign begin to overlap. With this potential we first derive the enthalpy $H_{n}(\sigma)$ of a kink chain containing $n$ kink pairs, taking account of the interaction of each kink with all the others. Due to the periodic nature of the Peierls potential, there exist, under the action of the same stress $\sigma$, a number of different kink chains with a different number n of kink pairs in equilibrium. We must therefore determine the 'ground state', which for given stress $\sigma$ is represented by the kink chain with $\mathrm{n}=\mathrm{k}$ kink pairs, having the lowest equilibrium enthalpy $\overline{\mathrm{H}}_{\mathrm{k}}(\sigma)$. This can only be done by numerical or semi-graphical methods. The numerical evaluation will be made for chains with up to $n=8$ kink pairs in dislocation segments between $\mathrm{L}=200 \mathrm{~b}$ and $10^{4} \mathrm{~b}$. The calculation can be extended to $\mathrm{n}>8$ in a straightforward way, but the computational effort increases with $n^{2}$. When deriving the enthalpy $H(\sigma)$ by a superposition of kink pairs, the long-range back-stress caused by the bow-out is not accounted 
for and will be considered separately. Finally we will discuss the asymmetry, which exists between forward and backward movement and the effects, which are expected under the action of a periodically varying stress.

\section{The kink pair.}

Calculating by analytical methods the equilibrium energy and equilibrium shape of a dislocation under the action of an applied stress is a formidable task. The general treatment requires a solution of an integro-differential equation and can only be performed by an iterative procedure [15]. When on the other hand the shape is known, the energy can be calculated [16] by performing line integrations along the dislocation line. The expressions for the elastic energy are however only valid, when the integration is performed over closed dislocation loops. Therefore it is generally not possible to determine the energy of a single kink, without defining the boundary condition. The expressions eqs (1a) and (1b) given above for the energy and shape of a single kink result from a local approximation, which manifests itself by the fact, that the cut-off radii $\Lambda$ and $r_{0}$ in the logarithm are ill defined. In contrast the energy of the kink pair has a well-defined meaning as the difference in energy with respect to a straight dislocation.

Since according to eq. 1 the kink approaches exponentially the Peierls valley, the configuration of the kink pair can to a good approximation be represented by a trapeze, where the straight side arms crossing the Peierls ridge have a projected length $\mathrm{w}$ on the $\mathrm{x}$-axis, and the length of the upper shorter segment is s. Fig. 1. The elastic energy of this configuration in [insert Fig. 1 about here]

anisotropic media was obtained by Püschl et al. [14], using a series expansion up to terms in $(a / w)^{2}$. With this result the energy $E_{K P}$ of a single kink pair can be derived [17] and is given by

with

$$
\begin{gathered}
\mathrm{E}_{\mathrm{KP}}=2 \mathrm{w} \Delta \mathrm{E}+\frac{\mathrm{a}^{2} \hat{\mathrm{T}}}{\mathrm{w}} \ln \left(\frac{\mathrm{w}}{\mathrm{r}_{\mathrm{o}}}\right)+\mathrm{V}_{\mathrm{KK}} \\
\mathrm{V}_{\mathrm{KK}}= \pm \frac{\mathrm{a}^{2} \hat{\mathrm{T}}_{\mathrm{L}}}{\mathrm{w}} \chi(\mathrm{p})
\end{gathered}
$$

and with

$$
\chi(p)=(1+p) \ln [1+p]-(1+p / 2) \ln [2+p]-(p / 2) \ln [p]
$$

where $\mathrm{p}=\mathrm{s} / \mathrm{w}$. The first term in eq. $5 \mathrm{a}$ is the increase in misfit energy for crossing the Peierls ridge. In sinusoidal potential we have $\Delta \mathrm{E}=\mathrm{E}_{\mathrm{P}} / 2$. The second term can be understood as the 
elastic self-energy $2 \mathrm{E}_{\mathrm{K}}$ of two isolated kinks widely separated, since for infinite separation $\mathrm{V}_{\mathrm{KK}}$ vanishes. The constant $\mathrm{r}_{0}$ is typically of the order of $\mathrm{b}$ and contains contributions from the elastic cut-off radius, the Kirchner integral and the variation in core energy. For details we refer to the original paper [14]. The term $\mathrm{V}_{\mathrm{KK}}$ is the interaction potential between the two kinks, with the '+' sign, when the kinks in the pair are of opposite sign and the '-'sign, when they are of the same sign. For $\mathrm{p}>>1$ the potential $\mathrm{V}_{\mathrm{KK}}$ reduces to the asymptotic value $\mathrm{U}_{\mathrm{KK}}$ of eq. 2. For $\mathrm{p} \rightarrow 0$ the potential $\mathrm{V}_{\mathrm{KK}}$ does however not diverge, and the value of $\mathrm{E}_{\mathrm{KP}}$ approaches the energy of a kink twice the height, when the kinks are of the same sign. For isolated kinks the equilibrium width, determined by the minimum of the energy, is given by

$$
\mathrm{w}_{0}=\mathrm{a} \sqrt{\frac{\hat{\mathrm{T}} \ln \left(\mathrm{w}_{0} / \mathrm{er}_{0}\right)}{\mathrm{E}_{\mathrm{p}}}}
$$

The equation for $\mathrm{w}_{0}$ can only be solved by (a rapidly converging) iteration. The corresponding energy of two kinks widely separated is then

$$
\mathrm{E}_{\mathrm{KP}}=2 \mathrm{a} \sqrt{2 \Delta \mathrm{E}_{\mathrm{L}} \ln \left(\mathrm{w}_{0} / \mathrm{er}_{0}\right)}\left(1+\frac{1}{2 \ln \left(\mathrm{w}_{0} / \mathrm{er}_{0}\right)}\right)
$$

With reasonable values of the parameters for metals we have $\mathrm{w}_{0}>>a$ and the value of the bracket is close to 1 . When, however, as in a kink chain, kinks of the same sign approach each other under the action of an applied stress to a separation of the order or less than $\mathrm{w}_{0}$, the equilibrium width can decrease considerably.

\section{The kink chain.}

With the self-energy $E_{K P}$ of a single kink pair and the Püschl interaction potential $V_{K K}$ we can now calculate the equilibrium configuration of a kink chain with a number of kink pairs by superposition. A general segment lying inclined to the direction of the Peierls valleys will also contain geometrical kinks and the expressions get very clumsy. We therefore limit the discussion here to segments originally along the $\mathrm{x}$-axis in a single Peierls valley, which contain an equal number of kinks of opposite sign The segments of length $L$ shall be anchored at $\mathrm{x}= \pm \mathrm{L} / 2$ and now bowed out under the action of an applied stress $\sigma$. We will assume that the acting stress is small compared to the Peierls stress $\sigma_{\mathrm{P}}$, so that the segments between the kinks will lie close to the bottom of the Peierls valleys. We will further assume, that the potential of the anchoring points is very short-ranged. We will consider shallow bowouts, which for a number $\mathrm{n}$ kink pairs implies $\mathrm{n} a<<\mathrm{L}$. 
The enthalpy $\mathrm{H}_{\mathrm{n}}(\sigma)$ of the chain is understood as difference to the enthalpy $\mathrm{H}_{0}$ of the straight segment without kinks. It is given by the self-energy $E_{S}$ of the $n$ kink pairs, the interaction energy $E_{I}$ of each of the $2 n$ kinks with all the others, and the work obA done by the applied stress $\sigma$, where A is the area swept over by the kink pairs. When $n$ kink pairs are formed, we have

$$
\mathrm{H}_{\mathrm{n}}(\sigma)=\mathrm{E}_{\mathrm{S}}+\mathrm{E}_{\mathrm{I}}-\sigma \mathrm{bA}
$$

where

$$
\begin{aligned}
& E_{S}=n\left(w E_{P}+\hat{T}_{L} \frac{a^{2}}{w} \ln \left(\frac{w}{r_{0}}\right)\right) \\
& E_{I}=\hat{T}_{L} \sum_{j>i}^{2 n} \frac{a_{i} a_{j}}{w} \chi\left(p_{i j}\right) \\
& A=a \sum_{i=1}^{n}\left(2 x_{i}+w\right)
\end{aligned}
$$

with the proviso

$$
\begin{gathered}
a_{i}=a_{j}=a \quad \text { for } 1 \leq \mathrm{i}, j \leq n, \quad a_{i}=a_{j}=-a \quad \text { for } n+1 \leq i, j \leq 2 n \\
x_{i}=-x_{2 n+1-i}, \quad x_{1}=-x_{2 n}=L / 2 \\
p_{i j}=\left(x_{j}-x_{i}-w\right) / w \quad \text { if } i, j \leq n \text { or } i, j \geq n+1 \text { and } p_{i j}=\left(x_{j}-x_{i}\right) / w \quad \text { if } i \leq n \text { and } j \geq n+1
\end{gathered}
$$

The equilibrium configuration and the equilibrium enthalpy $\bar{H}_{n}(\sigma)$ of the chain under the action of an applied stress $\sigma$ must now be determined by finding for each value of $\sigma$ the minimum of $\mathrm{H}_{\mathrm{n}}(\sigma)$ with respect to the positions $\left\{\mathrm{x}_{\mathrm{i}}\right\}$ of the different kinks and their width $\mathrm{w}$. Due to the geometrical restraints, enforced by the Peierls potential, for the same stress $\sigma$ however different bow-outs with different heights $\mathrm{h}=\mathrm{n} a$ can exist simultaneously. The problem is therefore to find the 'ground state', which is the bow-out with the lowest enthalpy.

The search for the ground state is somewhat involved and is best discussed by considering Fig. 2 obtained from the numerical evaluation made in the next section. We show there the [Insert Fig. 2 about here]

equilibrium enthalpies $\bar{H}_{n}(\sigma)$ of different kink chains with a different number $n$ of kink pairs formed in a segment of length $\mathrm{L}=200 \mathrm{~b}$ under the action of an increasing applied stress $\sigma$. When $\sigma$ is smaller than the Peierls stress $\sigma_{\mathrm{P}}$, the bow-out cannot form by mechanical action alone, but requires kink pair formation by thermal activation, which, as we assume, can occur readily. It will, however, only form when the enthalpy decreases, and therefore we need only 
to consider configurations with $\overline{\mathrm{H}}_{\mathrm{n}}(\sigma)<0$. This requires for instance as necessary but by no means sufficient condition $\sigma>\mathrm{n} \mathrm{E}_{\mathrm{KP}} / \mathrm{baL}$. Furthermore the stress must be large enough to overcome the mutual attraction of the kinks. For $n=1$ we have for instance $\sigma>\hat{T}_{L} a / 2 b L^{2}$.

When the number of kink pairs $n$ increases, the self-energy of the chain increases, but at the same time the applied stress $\sigma$ can do work in a larger area and hence the magnitude of the slope $\bar{H}_{n}(\sigma)$ vs. $\sigma$ will increase. Therefore the curves $\bar{H}_{n}(\sigma)$ for different $n$ will intersect. As we see for an increasing stress there will be for instance an interval $\sigma_{n}^{0} \leq \sigma \leq \sigma_{n}^{1}$ in which let say the chain with a number $\mathrm{n}=\mathrm{k}$ of kink pairs will have the lowest enthalpy and hence represents the ground state $\overline{\mathrm{H}}_{\mathrm{k}}(\sigma)$.With increasing stress at $\sigma_{\mathrm{k}}^{1}=\sigma_{\mathrm{k}+1}^{0}$ a new ground state $\overline{\mathrm{H}}_{\mathrm{k}+1}(\sigma)$ will be taken over by the kink chain with the number $\mathrm{n}=\mathrm{k}+1$ kink pairs in the interval $\sigma_{\mathrm{k}+1}^{0} \leq \sigma \leq \sigma_{\mathrm{k}+1}^{1}$. The chain with the original number of kink pairs still will be mechanically stable, though with the increased kink pair separation and a decreased kink width. With increasing stress an equivalent change-over will take place with an everincreasing number of kink pairs. The problem of describing the bow-out reduces now to the problem of finding the set $\left\{\sigma_{\mathrm{k}}^{0}\right\}$ where in the different ground states the number of kink pairs increases by 1 . The bow-out height of the chain, (determined by the equilibrium number of kink pairs in the ground state), will then be represented by a step function, where with increasing stress at each value of $\sigma_{\mathrm{k}}^{0}$ the height increases by $a$.

They are however two restrictions, which will be considered below: First. due to the geometrical restraints, the movement becomes asymmetric under the action of an increasing or decreasing stress and second, due to the bow-out an internal back-stress will build up, which will limit the bow-out height.

\section{Numerical evaluation.}

For the numerical evaluation we have chosen three dislocation segments of different total length $\mathrm{L}=200 \mathrm{~b}, 10^{3} \mathrm{~b}$ and $10^{4} \mathrm{~b}$ of original screw orientation, with prelogarithmic line tension factor $\hat{T}_{L}=\left(\mu b^{2} / 4 \pi\right)(1+v) /(1-v)$ using $v=1 / 3$. The line energies and enthalpies were normalised with $\mu \mathrm{b}^{2}$ and the stresses are given in units of $10^{-6} \mu$. For the Peierls energy we have chosen the values $E_{P}=5 \times 10^{-4} \mu b^{2}$ and $5 \times 10^{-5} \mu b^{2}$. For sinusoidal Peierls potentials the corresponding Peierls stress would be $\sigma_{\mathrm{P}}=\pi \mathrm{E}_{\mathrm{P}} / \mathrm{ab}$. The equilibrium widths of the kinks without applied stress for these two Peierls potential would be $\mathrm{w}_{0}=27 \mathrm{~b}$ and $\mathrm{w}_{0}=108 \mathrm{~b}$ resp. For the kink height we choose for simplicity $\mathrm{a}=\mathrm{b}$. Since for a chain with $\mathrm{n}$ kink pairs, each of 
the $2 n$ kinks interacts with all the others, the number of terms in the interaction energy increases with $2 n(2 n-1) / 2$, we consider here configurations with up to $n=8$. This is small enough to satisfy the condition na $<<\mathrm{L}$, but large enough to show the general trends. The search for the minimum, which only can be made by numerical methods, is somewhat involved. The interaction energy depends on the parameters $p_{i j}=\left(x_{j}-x_{i}-w\right) / w$ and with $a$ change in the applied stress $\sigma$, there will be simultaneous changes in $\left\{x_{i}\right\}$ and $w$. The numerical search for the minimum must therefore be made by an iterative procedure and when the starting values in the set $\left\{x_{i}, w\right\}$ are not chosen very close to the equilibrium values, the numerical procedure will become unstable and diverge.

For the segment with $\mathrm{L}=200 \mathrm{~b}$ and $\mathrm{E}_{\mathrm{P}}=5 \times 10^{-4} \mu \mathrm{b}^{2}$ the equilibrium enthalpies $\overline{\mathrm{H}}_{\mathrm{n}}(\sigma)$ for kink chains with number of kink pairs $1 \leq \mathrm{n} \leq 8$ are shown in Fig. 2, where for each value of $\{n, \sigma\}$ the equilibrium position of the different kinks $\bar{x}_{i}(\sigma)$ and their equilibrium width $\overline{\mathrm{W}}$ had to be determined. The minimal stress $\sigma_{\mathrm{k}-1}^{1}=\sigma_{\mathrm{k}}^{0}$, where the ground state is given by the configuration with $\mathrm{n}=\mathrm{k}$, can be determined graphically by the intercept of the curves of the enthalpy $\overline{\mathrm{H}}_{\mathrm{n}-1}(\sigma)$ and $\overline{\mathrm{H}}_{\mathrm{n}}(\sigma)$. For $\sigma=0$ the equilibrium width was $\mathrm{w}_{0} \approx 27 \mathrm{~b}$ but with increasing stress and bow-out height it decreases and in the ground state for $\mathrm{k}=8$ at the stress of $\sigma \approx 1.03 \times 10^{-3} \mu$ it has a value of $w \approx 7 b$. In Fig. 3 we show the resulting step-by-step [Insert Fig. 3 about here]

increase in bow-out height $h_{\mathrm{KC}}(\sigma)$ of the kink chain together with the continuous bow-out height $h_{\mathrm{LT}}(\sigma)$ in the line tension approximation (LTA), which is given by

$$
\mathrm{h}_{\mathrm{LT}}=\frac{\sigma b \mathrm{~L}^{2}}{8 \hat{\mathrm{T}}_{\mathrm{L}} \ln \left(\mathrm{L} / \mathrm{r}_{0}\right)}
$$

We see that under the conditions considered here the bow-out height in the ground state and in the LTA have about the same magnitude.

In Fig. 4 we show all the stable configurations of bow-outs with up to $n=8$ kink pairs [Insert fig. 4 about here]

which, according to the enthalpy $\overline{\mathrm{H}}_{\mathrm{n}}(\sigma)$, can exist in mechanical equilibrium at a stress of $\sigma$ $=6 \times 10^{-4} \mu$. This corresponds to the states represented by a section of the curves for $\bar{H}_{n}(\sigma)$ in Fig. 2 at this stress. The kinks were represented by their shape given by eq. 1, and the equilibrium width decreased from $\mathrm{w}_{0} \approx 27 \mathrm{~b}$ for $\mathrm{n}=1$ to $\mathrm{w} \approx 9 \mathrm{~b}$ for $\mathrm{n}=8$. The ground state for 
this stress exists for $n=4$. The representation in the figure is somewhat misleading, since in real space the height to length ratio must be reduced by a more than the factor of 100 . We mention here, however, as a caveat, that in our considerations the action of the global backstress of the configuration has not been considered. As shown below, this will make the chains with $\mathrm{n}=7$ and 8 unstable.

When trying to repeat these calculations for the same segment lengths $L=200 \mathrm{~b}$ but $\mathrm{a}$ Peierls energy $E_{P}=5 \times 10^{-5} \mu b^{2}$, the calculations fail, except for a single kink pair. The reason is, that the equilibrium length of a single kink $\mathrm{w}_{0}=108 \mathrm{~b}$ is already larger than half the segment length and hence additional kink pairs would just overlap. Under these conditions the description by a kink chain loses its meaning and the dislocation would only show 'ripples'..

For a segment of length $\mathrm{L}=10^{3} \mathrm{~b}$ the differences in the bow-out height between the kink chain and the LTA begin to show up. In Fig. 5 we show the increase in bow-out height $\mathrm{h}_{\mathrm{KC}}$ of [Insert Fig.5 about here]

the kink chain in the ground state for the Peierls energies $E_{P}=5 \times 10^{-4} \mu b^{2}$ and $5 \times 10^{-5} \mu b^{2}$ and $\mathrm{h}_{\mathrm{LT}}$ in the LTA for an increasing stress, and in Fig. 6 the same for the segment of $\mathrm{L}=10^{4} \mathrm{~b}$, where the differences become even more pronounced. In Fig. 7 we show the configurations of [Insert Fig.6 and Fig.7 about here]

the bow-outs, for a Peierls energy $E_{P}=5 \times 10^{-4} \mu b^{2}$ in the segment of $L=10^{4} \mathrm{~b}$. At a stress where the first kink pair can be formed, the bow-out in the LTA (marked '0') would have reached the height of $\mathrm{h}_{\mathrm{LT}} \approx 29 \mathrm{~b}$ and at a stress where the kink chain in the ground state (marked ' $k 1$ ') would have reached the bow-out height of $8 \mathrm{~b}$, the bow-out in the LTA (marked ' 1 ') would have reached the value of $\mathrm{h}_{\mathrm{LT}} \approx 36 \mathrm{~b}$. (The states ' $\mathrm{k} 2$ ' and ' $\mathrm{k} 3$ ' can only be reached in a reverse cycle as discussed below).

As can be seen, however, the average slope $h_{\mathrm{KP}}(\sigma) \mathrm{Vs} \sigma$ for stresses $\sigma$ above the critical stress $\sigma_{\mathrm{cr}}$, the height of the kink chain increases nearly the same amount as the bow-out of the LTA. Hence the kink chain, when bowing out in the forward direction, has nearly the same 'elastic stiffness' as a normal dislocation segment without kinks.

\section{The back stress}

We have obtained the enthalpy $\bar{H}_{n}(\sigma)$ of the bow-out from the superposition of pair potentials. By this procedure we implicitly assume that all the kinks are projected into the $\mathrm{x}$ axis and move only laterally. Though with $\mathrm{n} a<<\mathrm{L}$ the bow-out is very shallow, the influence of the movement perpendicular to the $\mathrm{x}$-axis cannot be neglected, since it produces a long- 
range back stress $\sigma_{\mathrm{b}}$ counteracting the applied stress. This back stress, acting at the leading segment, can in principle be obtained a by integrating the stress exerted by all he line elements along the side branches of the dislocation line, which would be a very tedious procedure. A global estimate of the back stress may however be obtained when the approximate the shape by a pair of super-kinks of height $\mathrm{n} a$ and width $\mathrm{w}_{\mathrm{s}} \approx \mathrm{nw}$. The back stress resulting from these two side branches will not be homogeneous and will be largest close to the ends of the leading segment. In order that the configuration is stable, the local effective stress $\sigma_{\text {eff }}(\mathrm{x})=\sigma-\sigma_{\mathrm{b}}(\mathrm{x})$ it must be balanced by the Peierls stress. An upper limit for the stability of the bow-out is reached, when the effective stress at the leading segment becomes negative everywhere, which implies that it must vanish. at the centre of the leading segment at $\mathrm{x}=0$. For this configuration made up by straight segments, the stress can be obtained in closed form [16] and we obtain

$$
\sigma_{\mathrm{b}}(0)=\frac{2}{\left(\mathrm{~L} / 2-\mathrm{w}_{\mathrm{s}}\right) \sin \gamma}\left[-\mathrm{E}_{\mathrm{L}}(\Theta) \cos (\Theta-\gamma)+\frac{\mathrm{dE}_{\mathrm{L}}}{\mathrm{d} \Theta} \sin (\Theta-\gamma)\right]_{\Theta_{1}}^{\Theta_{2}}
$$

with $\gamma=\arctan \left(n a / w_{\mathrm{s}}\right)$, and for screw dislocations $\Theta_{1}=\arctan \left(\mathrm{na} /\left(\mathrm{L} / 2+\mathrm{w}_{\mathrm{s}}\right)\right), \Theta_{2}=0$.

We have now evaluated the back stress for the configurations shown in Fig. 4, which according to the enthalpy calculations represent all the states up to $n=8$, which would be mechanically stable at an applied stress $\sigma_{\mathrm{a}}=6 \times 10^{-4} \mu$ In Fig. 8 we show the resulting [Insert Fig. 8 about here]

effective stress $\sigma_{\text {eff }}=\sigma-\sigma_{\mathrm{b}}(0)$ for the various kink chains together with the Peierls stress $\sigma_{\mathrm{P}} \cong$ $\pi \mathrm{E}_{\mathrm{P}} / \mathrm{ba}$. The ground state of this configuration would be for $n=4$. The effective stress is balanced by the Peierls stress at all positions. However for the configurations with $n>6$ the effective stress becomes negative everywhere along the leading segment and the corresponding kink chains would be unstable. This is a rather crude estimate and a more detailed but rather involved calculation of the back-stress would probably reduce the accessible height of the kink chain even more.

\section{Periodic stress variations.}

Up to now we have considered the mechanical stability of kink chains under the action of a static applied stress, which may be of increasing magnitude. Since the energy of the configurations with different number of kinks are separated by energy barriers, the ground state can only be reached, when thermal activation can take place. The dynamic behaviour of the kink chain under a varying stress can therefore be described adequately only by a treatment based on thermodynamics of irreversible processes and the problem is aggravated 
by the fact, that not even a steady state exists. We use here a mechanistic approach and consider thermal activation only as means to reach mechanical equilibrium. This will however introduce an asymmetry in the movement, depending on whether the external applied stress increases or decreases. Formation of a kink pair by thermal activation can occur only in the forward direction. Thermal activation against a decreased but still positive stress could not create a stable pair, since the driving force acting at the kinks will tend to shrink the newly formed pair and no saddle point exists. Therefore the backward movement will occur by kink pair collapse. Kink pair collapse can take place even spontaneously, when the applied stress $\sigma$ or rather the effective stress $\sigma_{\text {eff }}$ has decreased to a value too small to counteract the mutual attraction of the two kinks in the leading kink pair. This will occur when the equilibrium separation in the pair decreases below a critical value $L_{c r}=\sqrt{\hat{T}_{L} a / 2 b \sigma_{\text {eff }}}$.

The behaviour of the segment upon stress reversal now depends sensitively on the length of the segment. As seen in Fig. 3, for a segment of $L=200 \mathrm{~b}$ and the value of the Peierls energy $E_{P}$ chosen, the bow-out height in the kink chain and the corresponding about height in the LTA are approximately the same. Therefore, as discussed above, the back stress will have decisive influence and induce the back movement.

The situation is different in the segment of $\mathrm{L}=10^{4} \mathrm{~b}$ for which the configuration is shown in Fig. 7. At the stress of $\sigma \approx 4.3 \times 10^{-6} \mu$, where the ground state would have reached a bowout of height 8b (marked ' $k 1$ '), the corresponding bow-out in the LTA (marked '1') would have a height $\mathrm{h}_{\mathrm{LT}} \approx 36 \mathrm{~b}$. The back- stress caused by the side branches in the configuration $\mathrm{k} 1$ is much too small to have any influence on the leading segment. When now upon stress reversal the applied stress has receded to a value of $\sigma \approx 0.93 \times 10^{-6} \mu$, where based on the LTA we would obtain a bow-out (marked ' 2 ') of height of $\mathrm{h}_{\mathrm{LT}} \approx 8 \mathrm{a}$ the back-stress produced by the side branches in the corresponding kink chain (marked ' $\mathrm{k} 2$ ') is still too small to induce backward movement and hence the kink chain would still remain in its original configuration. When the decreasing external stress reaches finally a value of $\sigma \approx 0.22 \times 10^{-6} \mu$, which in the LTA would give $\mathrm{h}_{\mathrm{LT}} \approx 1.8 \mathrm{~b}$ (marked ' 3 '), the separation of the kinks in the leading segment (marked 'k3') would approach the critical distance, where spontaneous collapse takes place.

This asymmetry in the movement introduces some special effects under dynamic conditions, when the applied stress changes periodically. Whereas the forward movement is always thermally activated, the back movement in short segments is induced by the backstress, and will be in phase with the applied stress. This can give rise to a dynamic hysteresis, with the resulting energy dissipation. For long segments, however, the bow-out of the kinks 
will remain at its maximum height, until the applied stress has returned to a small value. This 'ratchet-effect' will gives rise to a static hysteresis, independent of temperature and frequency. The situation is even more complicated when there exist intermediate situations, where the segments are under an action of a static pre-stress. This energy dissipation under periodic conditions leads to internal friction effects, which can be observed experimentally and which, for instance in fcc metals, are known as Bordoni relaxation [18].

\section{Discussion.}

We have studied the bow-out of dislocation segments in crystalline solids, lying originally along close-packed lattice directions, under the action of an applied stress. The segments were assumed to be pinned a distance $\mathrm{L}$ apart in the same Peierls valley. For stresses smaller than the Peierls stress, the bow-out will occur by kink pair formation across the ridges of the Peierls potential. The kink pairs were represented by piece-wise straight segments, having the shape of a trapeze, which gives a reasonably good approximation to their shape and a very good approximation to their self and interaction energies.

The equilibrium configuration of a kink chain consisting out of $n$ kink pairs was determined by minimizing its enthalpy $\mathrm{H}_{n}(\sigma)$, taking account of the interaction energy of each kink with all the others. Since under the action of an applied stress the kinks of the same sign approach each other very closely, the usual asymptotic $1 / \mathrm{r}$ interaction potential cannot describe the situation correctly, since it diverges for small separations. We therefore used the more realistic Püschl potential [14] which approaches the energy of a kink twice the height, when two kinks of the same sign begin to overlap. Under the action of the applied stress the kinks not only change their positions but also their width, and when the kinks of same sign approach each other in the side branches, the width decreases below the one of isolated kinks.

From a thermodynamic point of view we would actually have to consider the change in free enthalpy $\Delta \mathrm{G}_{\mathrm{n}}=\Delta \mathrm{H}_{\mathrm{n}}-\mathrm{T} \Delta \mathrm{S}_{\mathrm{n}}$. where $\Delta \mathrm{S}_{\mathrm{n}}$ is the change in entropy of the system. The entropy change $\Delta S$ has been discussed in detail by Seeger and Schiller [5,8] and Alefeld [9] and has two contributions: the change in 'self entropy', which results from the change in the vibrational spectrum of the dislocation when a kink pair is introduced, and the 'interaction entropy' which results forms the change in vibrational spectrum of the kinks, which are coupled by long-range forces. By including these terms and considering the influence of the temperature would add considerable complexity to an already very complex problem. By neglecting the entropy term, our treatment actually corresponds to the situation at $\mathrm{T}=0$. 
For a fixed stress $\sigma$ above a critical stress there can exist in mechanical equilibrium a different number of kink chains with different numbers $n$ of kink pairs and hence discrete bow-out heights n $a$. On the other hand for a fixed bow-out height n $a$, there exists a continuum of mechanically stable configurations within a wide range of the applied stress $\sigma$. It is therefore of interest to find the ground state, which within a certain range of stress is represented by the configuration with $\mathrm{n}=\mathrm{k}(\sigma)$ kink pairs, having the lowest enthalpy in an ensemble of dislocation segments of length $\mathrm{L}$. This represents the configuration in thermodynamic equilibrium.

Since the kink chains with different number of kinks are separated by energy barriers, the ground state is only accessible, when kink pair formation can take place by thermal activation and the kinks can move to their equilibrium positions. Therefore the bow-out formation will show a dependence on temperature and strain rate. Under the condition of cyclic loading this can lead to a phase-lag between stress and anelastic strain and hence to an energy dissipation. In the reverse cycle kink pair formation in the backward direction against the decreasing but still positive stress cannot take place, since no saddle point exists. As result there will be an asymmetry: forward movement must occur at by thermally activated kink-pair formation, backward movement will occur by kink pair collapse, which can take place even without thermal activation. The resulting 'ratchet effect' can lead to a static hysteresis.

The stress $\sigma_{0}$, where the first kink pair can be formed mechanically, is determined by the condition $\mathrm{H}\left(\sigma_{0}\right) \leq 0$ and is given by $\sigma_{1}=\mathrm{E}_{\mathrm{KP}} / \mathrm{abL}$. On the other hand the bow-out height $\mathrm{h}_{\mathrm{LT}}$ in the LTA given by eq. 9 increases proportional to $\sigma \mathrm{L}^{2}$. The bow-out height $\mathrm{h}_{\mathrm{LT}}$ in the LTA at the stress $\sigma=\sigma_{1}$, where the first kink pair is formed is then given by

$$
\mathrm{h}_{\mathrm{LT}}^{0}=\frac{\mathrm{E}_{\mathrm{KP}} \mathrm{L}}{8 \mathrm{a} \hat{\mathrm{T}} \ln \left(\mathrm{L} / \mathrm{r}_{0}\right)} \approx \frac{1}{4} \sqrt{\frac{\mathrm{E}_{\mathrm{p}}}{\hat{\mathrm{T}}_{\mathrm{L}} \ln (\mathrm{L} / \mathrm{w})}} \mathrm{L} .
$$

Therefore the discrepancy in the bow-out configuration between the kink chain and the LTA increases for larger segment lengths $L$. In our example with a Peierls energy of $E_{P}=5 \times 10^{-4} \mu b^{2}$ the bow-out height in segments of $\mathrm{L} \approx 200 \mathrm{~b}$ in the two models agreed roughly, but in longer segments the differences can become very pronounced, as can be seen in Fig. 7. At the stress where the smooth bow-out in the LTA of parabolic (or circular) shape would have reached a value of about $36 \mathrm{~b}$, the equilibrium configuration of the kink chain in the ground state would be of trapezoidal shape with height $8 \mathrm{~b}$. The fact that in TEM observations in metals, segments of this length at room temperature can show a smoothly curved shape, indicate that at this temperature the Peierls energy must be extremely low. At low temperatures 
dislocations remain smoothly curved in fcc metals, but dislocations in bcc metals show long straight segments arranged in screw orientation, in spite of the fact that the segments are under the action of internal stresses. This would be expected, when the dislocations take up the ground state in the presence of a larger Peierls energy. The situation exists even at elevated temperatures in crystals with covalent binding such as semiconductors, where long straight dislocation segments either in screw- or $60^{\circ}$ - orientation are observed.

When deriving the enthalpy $\mathrm{H}_{n}(\sigma)$ of the kink chain by a superposition of $n$ pair interactions, we have neglected the fact that by the bow-out a long-range opposing back stress is produced. The action of the back-stress could be accounted for, when the equilibrium configuration is obtained by numerical simulation methods based on the balance of stress [19], which by now have reached a high degree of sophistication. Since however for each stress there exist a number of configurations with different bow-out heights, all in mechanical equilibrium, it would be difficult to identify the ground state with the lowest energy on the basis of stress balance. We have made here a crude estimate of the back stress and shown, that it can limit the bow-out height of the kink chain, when it approaches the height resulting from the line tension approach, especially in segments of small length. A more detailed calculation of this back stress would be, however, very desirable and it will further reduce the accessible number of stable configurations.

\section{References :}

[1] R. Peierls, Proc, Phys. Soc. London 52 , 34 (1940).

[2] F. R. N. Nabarro, Proc. Phys. Soc. London 59, 256 (1947).

[3] W. Shockley, Trans. AIME 194, 829 (1952).

[4] J. Frenkel and T. Kontorova, Phys. Z. Sowjet. 13, 1 (1938)

[5] A. Seeger and P. Schiller, Acta Met. 10, 348 (1962).

[6] F. Kroupa and L. M. Brown, Phil. Mag. 6, 1267 (1961).

[7] J. D. Eshelby, Proc. Roy. Soc. 266 A, 222 (1962).

[8] A. Seeger and P. Schiller, In: Physical Acoustics (P. Mason ed.), Academic Press, vol. III A, 1966, p.361.

[9] J. Alefeld, Appl. Phys., 36, 2633 (1965).

[10] W. Lems, Physica 30, 1617 (1964).

[11] R. J. Arsenault, Acta Metall. 15, 501 (1967).

[12] C. Esnouf and G. Fantozzi, G, Phys. Stat. Sol. A 47. 201 (1978). 
[13] M. Bujard, G. Gremaud and W. Benoit, J. Appl. Phys. 62, 3173 (1987).

[14] W. Püschl, G. Schoeck and H. O. K. Kirchner, Phil. Mag. 56, 553 (1987).

[15] H. O. K. Kirchner, Phil. Mag A 43, 1393 (1981).

[16]J. P. Hirth and J. Lothe, Theory of Dislocations, Wiley, New York, 1982.

[17] G. Schoeck and W. Püschl, phys. stat. sol. (b) 144, 105 (1984).

[18] G. Fantozzi, C. Esnouf, W. Benoit and I. G. Ritchie, Prog. Mater. Sci. 27, 211 (1982).

[19] K. W. Schwarz, J. Appl. Phys 85, 108 (1999). 


\section{Figure Captions.}

Fig. 1:

Configuration of kink-pairs. The kink width is $\mathrm{w}$, and the lengths of the straight segment between two kinks is defined as $\mathrm{s}$. The position of the kinks are defined by the coordinate $\mathrm{x}_{\mathrm{i}}$ of their upper end.

Fig. 2:

Equilibrium enthalpy $\overline{\mathrm{H}}(\sigma)$ of kink-chains in a dislocation segment in screw orientation with length $L=200 \mathrm{~b}$ as function of stress for chains with up to $\mathrm{n}=8$ kink-pairs. The Peierls energy is assumed to be $E_{P}=510^{-4} \mu b^{2}$ and the corresponding Peierls stress is $\sigma_{\mathrm{P}} \approx 1.510^{-3} \mu$. The ground state is marked when the corresponding number $\mathrm{n}=\mathrm{k}$.

Fig. 3:

The bow-out height $\mathrm{h}$ for the kink-chain in the ground state and in the LTA for a dislocation segment of in screw orientation with length $L=200 \mathrm{~b}$ as function of applied stress. The Peierls energy is $E_{P}=510^{-4} \mu b^{2}$ and the corresponding Peierls stress is $\sigma_{\mathrm{P}} \approx 1.510^{-3} \mu$

Fig. 4:

Equilibrium configurations derived from the kink-kink interactions of different kinkchains in a dislocation segment of in screw orientation with length $L=200 \mathrm{~b}$ with up to $n=8$ kink-pairs under the action of a stress of $\sigma=610^{-4} \mu$. The ground state is for $n=4$, and the corresponding bow-out in the LTA is also shown

Fig. 5:

The bow-out height $\mathrm{h}$ for kink-chains in the ground state and in the LTA for a dislocation segment of a screw dislocation with length $\mathrm{L}=10^{3} \mathrm{~b}$ as function of applied stress. The Peierls energy is assumed to be (1) $\mathrm{E}_{\mathrm{P}}=510^{-4} \mu \mathrm{b}^{2}$ and (2) $\mathrm{E}_{\mathrm{P}}=510^{-5} \mu \mathrm{b}^{2}$

Fig. 6:

The bow-out height $\mathrm{h}$ for kink-chains in the ground state and in the LTA for a dislocation segment of a screw orientation with length $\mathrm{L}=10^{4} \mathrm{~b}$ as function of applied stress. The Peierls energy is assumed to be (1) $E_{P}=510^{-4} \mu b^{2}$ and (2) $E_{P}=510^{-5} \mu b^{2}$ 
Fig. 7:

Equilibrium configurations of a kink-chain with $n=8$ kink-pairs for a segment in screw orientation and length $\mathrm{L}=10^{4} \mathrm{~b}$ under different applied stresses: $(\mathrm{k} 1)$ ground state for $\sigma \approx 4.3$ $10^{-6} \mu ;(\mathrm{k} 2) \sigma \approx 0.9310^{-6} \mu$ and. $(\mathrm{k} 3) \sigma \approx 0.2210^{-6} \mu$. The corresponding bow-outs in the LTA a marked with (1), (2) and (3) resp. The configuration marked (0) is the bow-out in the LTA for $\sigma \approx 3.310^{-6} \mu$, where the first kink pair is formed. The states $(\mathrm{k} 2)$ and $(\mathrm{k} 3)$ can only be reached in a reverse cycle, since they have a higher enthalpy than the ground states for the corresponding stresses.

Fig. 8:

The effective stress $\sigma_{\text {eff }}=\sigma-\sigma_{\mathrm{b}}$ at the center $\mathrm{x}=0$ of the leading segment for the kinkchains shown in Fig. 4. The effective stress is negative for the chains with more than $n=6$ kink-pairs. Hence the chains with $n=7$ and 8 will not be stable. 


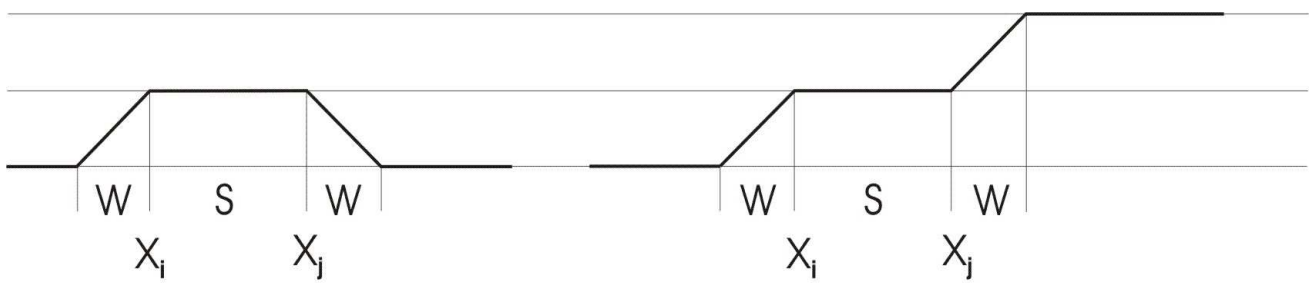

Fig. 1

$143 \times 43 \mathrm{~mm}(300 \times 300 \mathrm{DPI})$ 


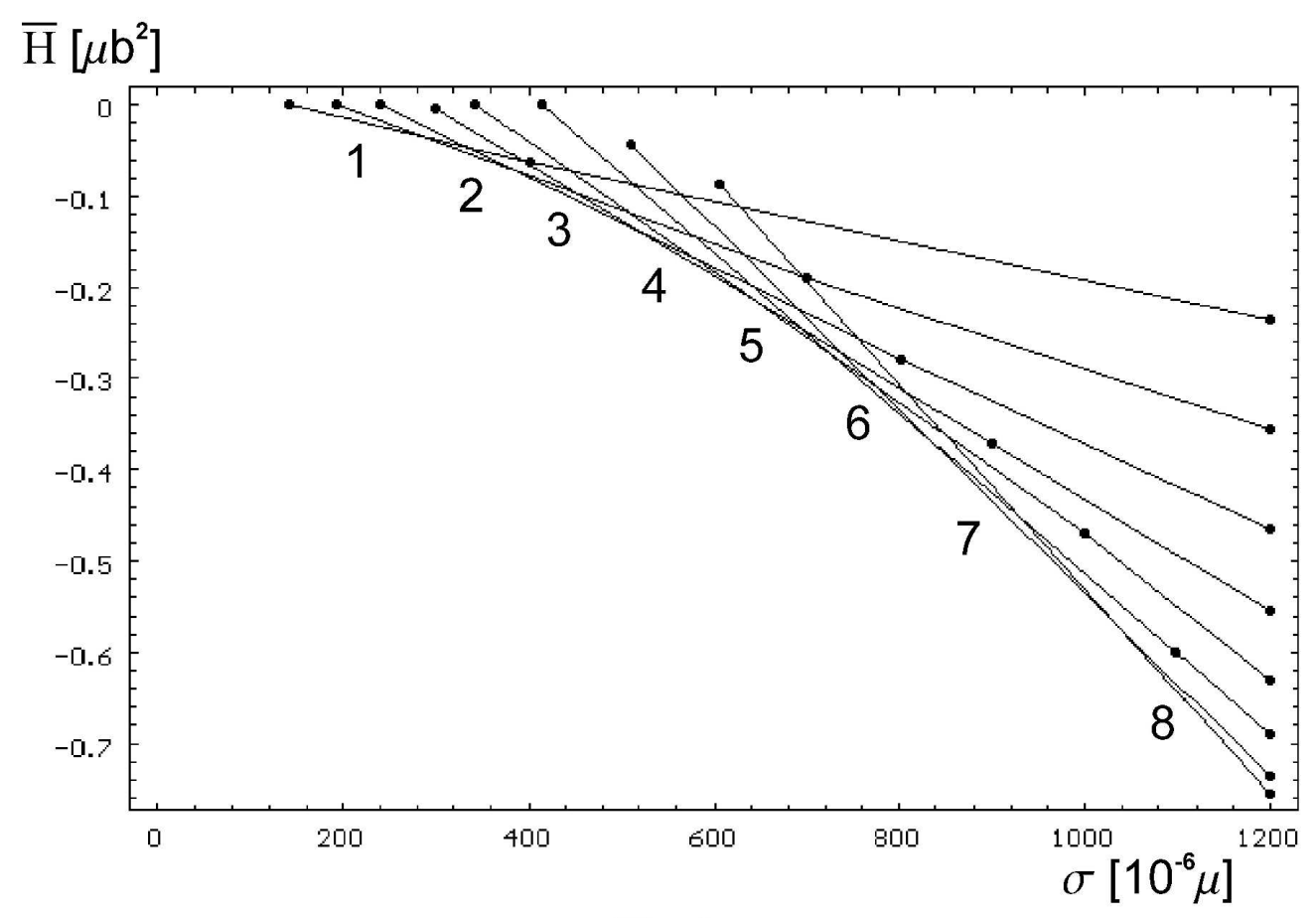

Fig.2

$1910 \times 1444 \mathrm{~mm}(72 \times 72 \mathrm{DPI})$ 


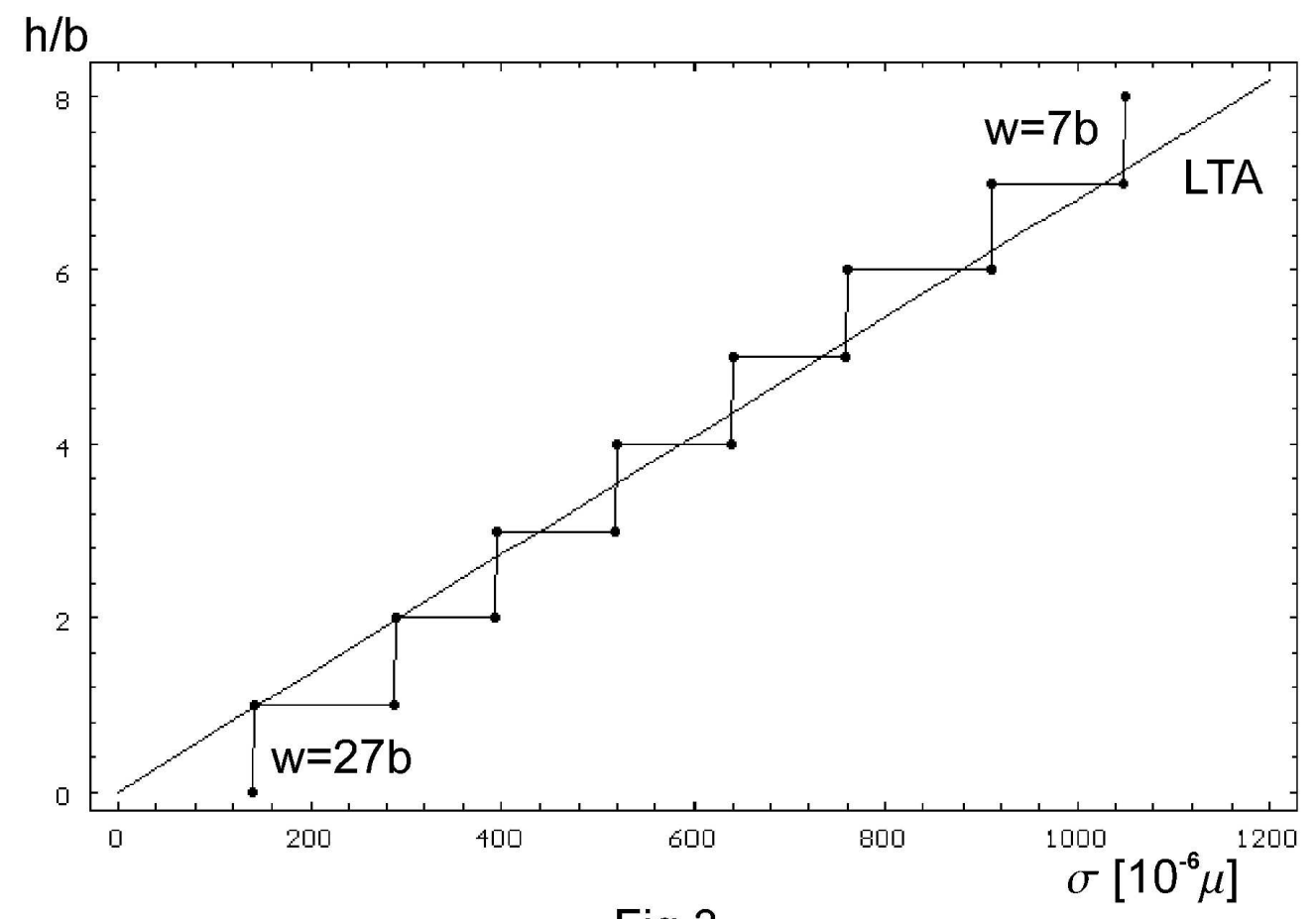

Fig.3

$1910 \times 1444 \mathrm{~mm}(72 \times 72 \mathrm{DPI})$ 


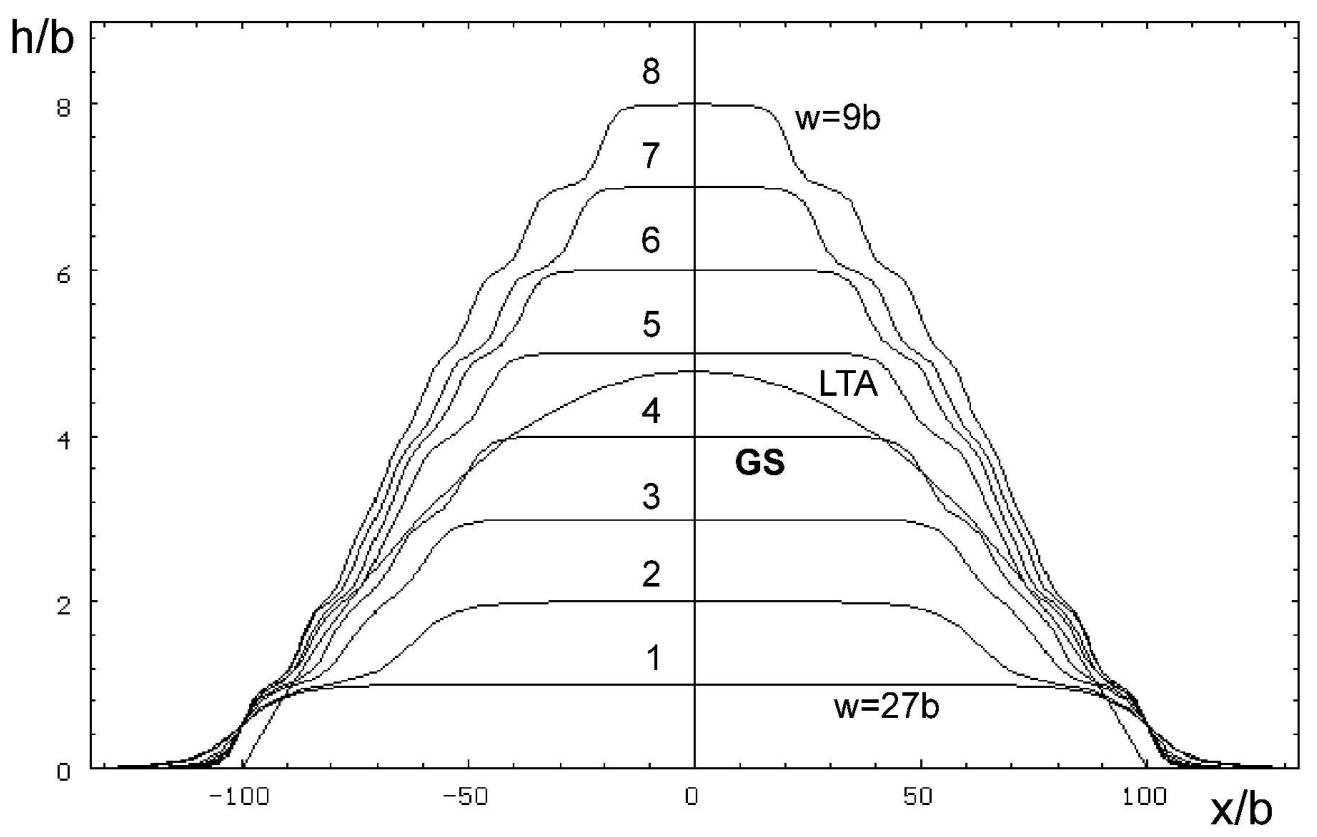

Fig.4

1932×1444mm (72 x 72 DPI) 


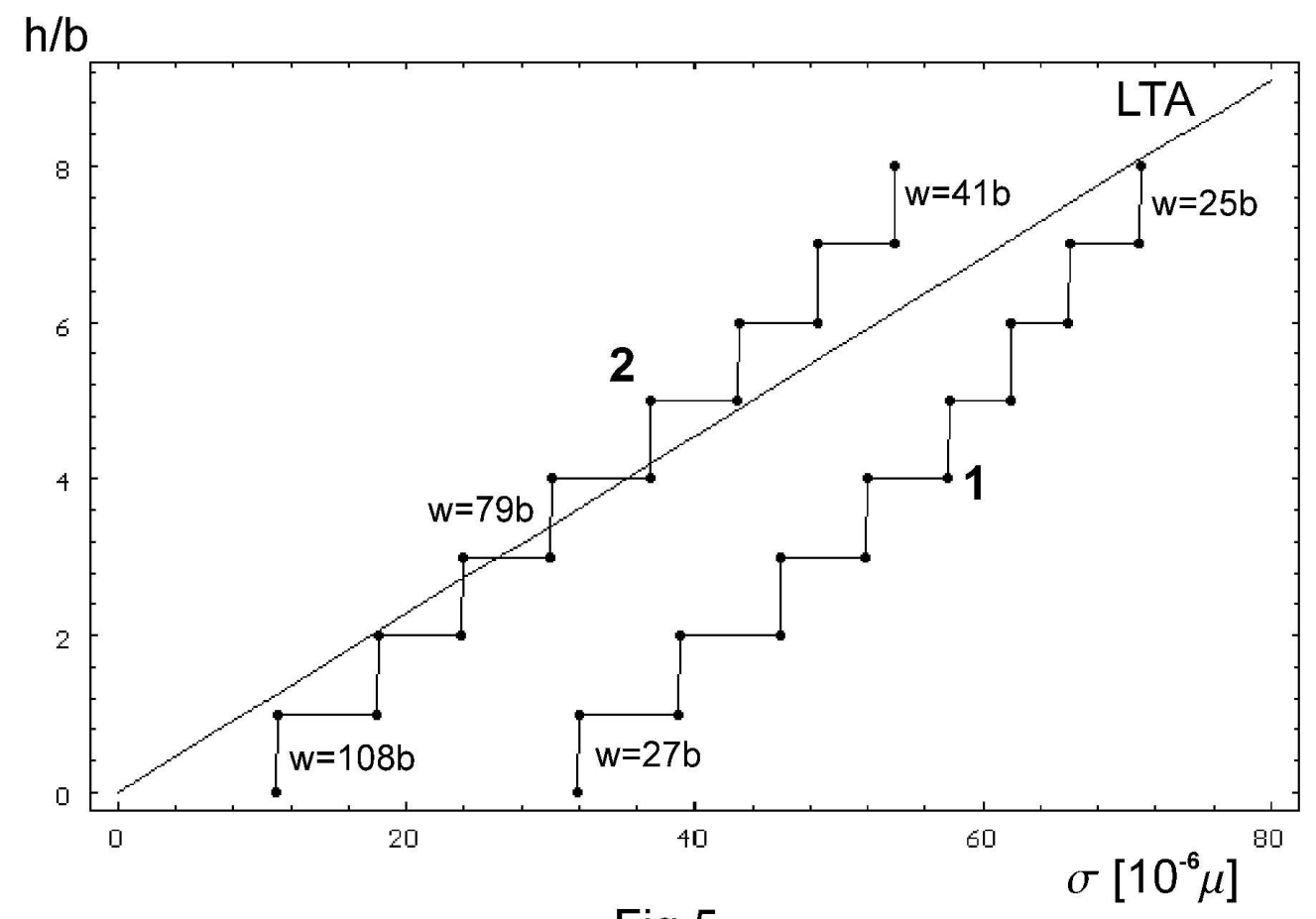

Fig.5

$1910 \times 1444 \mathrm{~mm}(72 \times 72 \mathrm{DPI})$ 


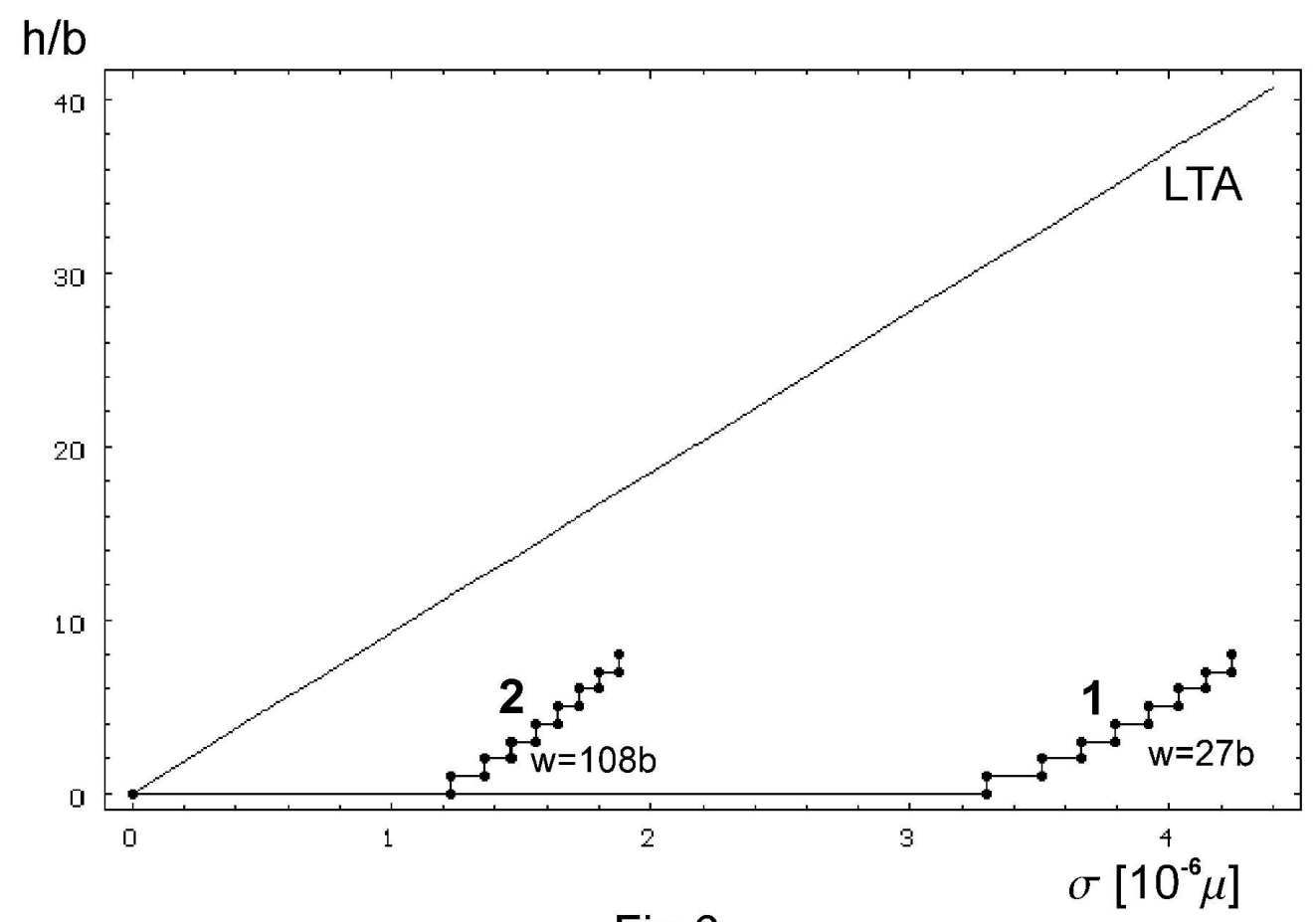

Fig.6

$1910 \times 1444 \mathrm{~mm}(72 \times 72 \mathrm{DPI})$ 


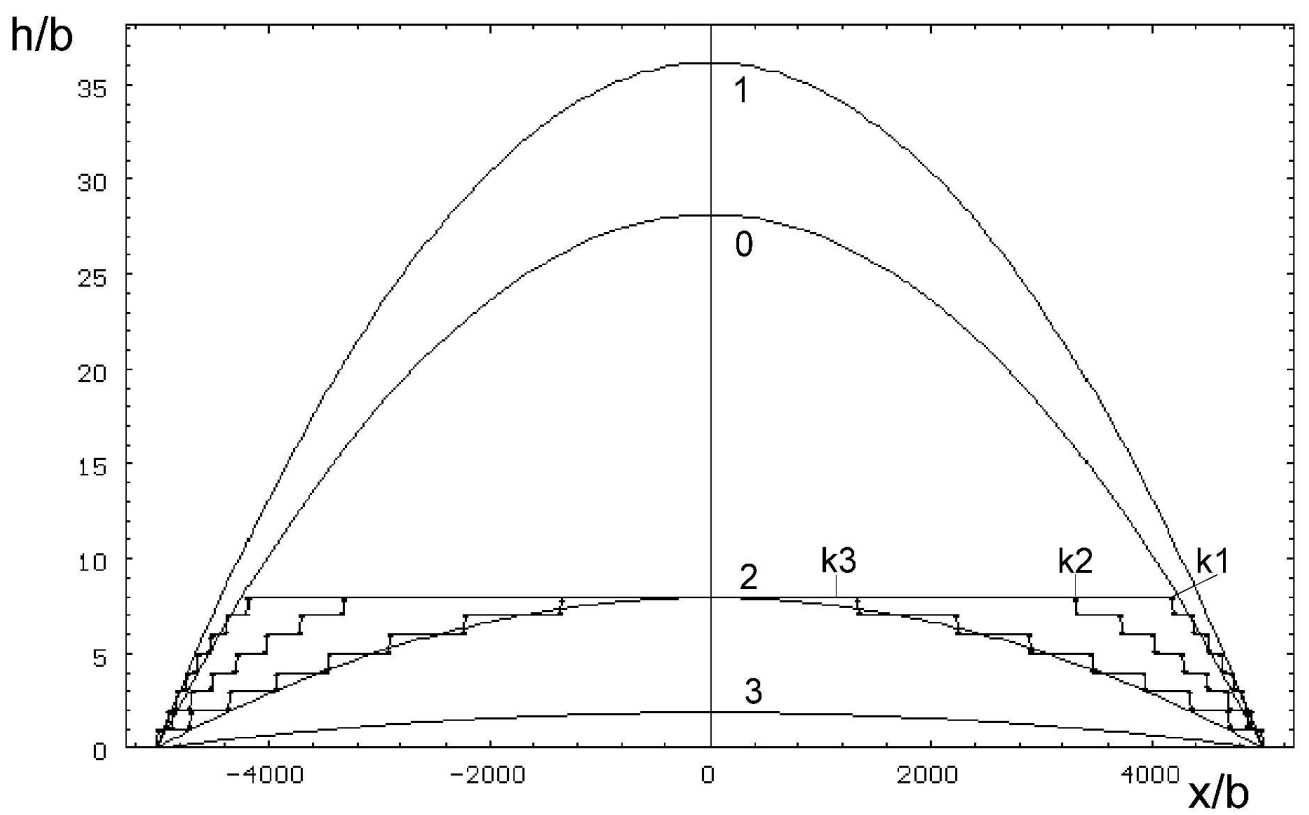

Fig.7

1963×1443mm (72 x 72 DPI)

http://mc.manuscriptcentral.com/pm-pml 
Page 25 of 25

Philosophical Magazine \& Philosophical Magazine Letters

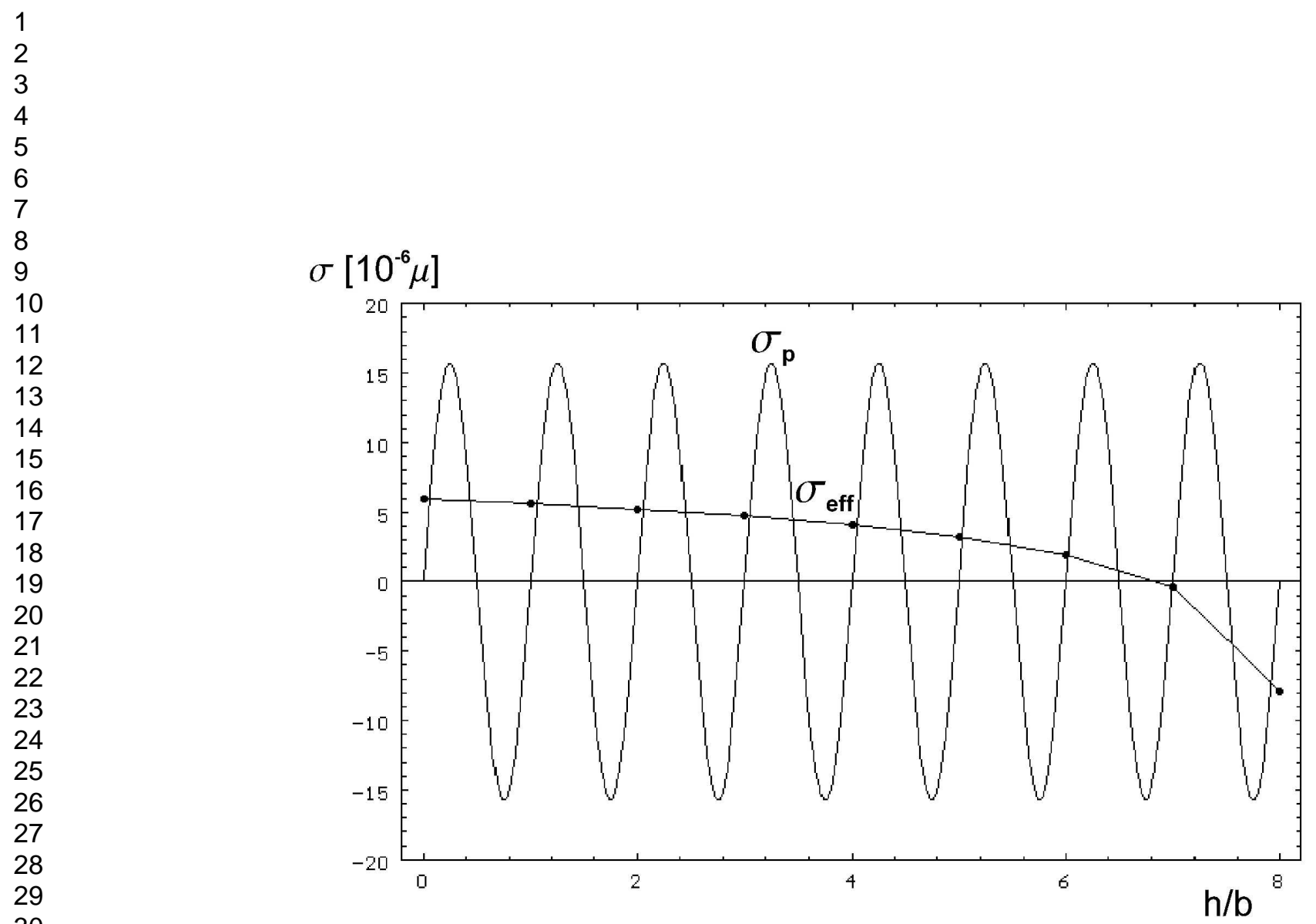

Fig .8

$1945 \times 1444 \mathrm{~mm}(72 \times 72$ DPI $)$

http://mc.manuscriptcentral.com/pm-pml 